\title{
REDUCCIÓN DE LA CARGA DE TRABAJO DEL OPERADOR EN UN CENTRO DE CONTROL ADAPTATIVO MULTI-VEHÍCULO
}

\author{
Juan A. Bonache-Seco, José A. López-Orozco, Eva Besada-Portas, \\ Juan F. Jiménez-Castellanos, José M. Girón-Sierra \\ Departamento de Arquitectura de Computadores y Automática. Universidad Complutense de Madrid (UCM) \\ \{jabonache, jalopez, ebesada\}@ucm.es, juan.jimenez@fis.ucm.es, gironsi@dacya.ucm.es
}

\begin{abstract}
Resumen
El incremento de los vehículos autónomos, la multitud de datos a tener en cuenta y el aumento de la complejidad de las tareas que realizan hace que un sólo operador tenga dificultades para monitorizar y controlar todos los vehículos de una misión. Aprovechando el diseño arquitectonico modular, versátil, reconfigurable y multi-vehículo del Centro de Control desarrollado, se han incorporado en el mismo mecanismos de ayuda (adaptabilidad y transparencia) que aligeran la información mostrada y se centran en aquella más relevante para cada situación dentro de una misión, con el fin de reducir la carga mental de trabajo del operador. Este artículo muestra cómo se han implementado estas ayudas y los experimentos realizados sobre un conjunto de operadores para verificar su eficacia en la reducción de su carga mental de trabajo.
\end{abstract}

Palabras clave: Carga mental de trabajo, Centro de control de tierra, Interfaces software adaptativas.

\section{INTRODUCCIÓN}

En la actualidad, la proliferación de vehículos autónomos y su uso para la realización de tareas colaborativas hace que se desarrollen centros de control capaces de gestionar varios vehículos simultáneamente, lo que implica mostrar al operador mucha información y que éste tenga que atender a un mayor número de indicadores para verificar la correcta evolución de la misión. Por este motivo, los desarrolladores de los Centros de Control de Tierra (CCT) se enfrentan al difícil reto de diseñar interfaces sencillos y ergonómicos, y que sean capaces de manejar simultáneamente varios vehículos o dispositivos del mismo tipo $[13,14]$ o diferentes $[7,10]$. El problema se agrava cuando los vehículos son heterogéneos y/o puede cambiar su número durante una misión.

El desarrollo de un CCT distribuido que soporte la carga computacional que implica supervisar múltiples vehículos heterogéneos y que además sea robusto, flexible, escalable, y adaptable en tiempo real implica numerosos retos tanto desde el punto de vista de la ingeniería y diseño de la arquitectura software, como de la investigación y aplicación de nuevas técnicas y paradigmas de programación que permitan implementar este tipo de software. Ejemplo de ello son el desarrollo de CCTs basados en arquitecturas reutilizables gracias a su estructura modular orientada a objetos y uso de patrones software [11], o arquitecturas centradas en modelos basados en grafos adaptables que permiten añadir o modificar módulos en tiempo real [1].

Otro aspecto importante a tener en cuenta al incorporar en el CCT múltiples vehículos es que la cantidad de datos que deben ser monitorizados y controlados por parte del operador para supervisar, y en caso necesario actuar, se multiplica. Esto incrementa enórmemente su carga mental de trabajo (Mental WorkLoad, WL) y estrés, provocando un descenso de su rendimiento, una mayor fatiga y un aumento de la probabilidad de que se produzca una negligencia o distracción [6]. Sin embargo, se ha observado que reducir sin más la información ofrecida al operador para paliar la avalancha de datos puede causar la falta de información suficiente para tomar una decisión, aumentando su estrés y desconfianza en el software [9].

Esto implica que es necesario encontrar formas de ofrecer la información al operador de forma adecuada (accesible, ergonómica, ...) y dependiente de la situación (adaptabilidad), y mostrar sólo la información necesaria para realizar sus tareas sin que aumente su desconfianza (transparencia). Trabajos como $[5,12]$ muestran que es posible aplicar la transparencia para reducir la carga mental de trabajo y el tiempo de respuesta del operador, y aumentar su rendimiento.

Por tanto, no sólo es aconsejable diseñar un CCT que sea adaptable a diferentes misiones y vehículos en tiempo real, sino que se debe procurar aprovechar el diseño versátil y reconfigurable para implementar métodos que muestren la información 
al usuario de forma adecuada, adapten la interfaz gráfica de usuario (Graphical User Interface, GUI) a las necesidades de la misión, y mejoren la carga de trabajo y rendimiento del operador.

La organización del artículo es la siguiente. En la sección 2 se describe el diseño arquitectónico de un CCT versátil y adaptable (frente a diferentes misiones y vehículos), y de su GUI adaptable mediante la aplicación de los conceptos de adaptabilidad y transparencia con el objetivo de reducir la carga de trabajo del operador. En la sección 3 se presenta el marco experimental y los criterios utilizados para evaluar la versatilidad del CCT diseñado y su GUI. En la sección 4 se presentan los resultados obtenidos en el proceso de evaluación de un conjunto de individuos, y se determina que la propuesta es un método eficaz para reducir la carga de trabajo del operador. Finalmente, en la sección 5 se exponen las conclusiones del trabajo realizado y algunas lineas de trabajo futuro.

\section{EL CCT ADAPTATIVO}

Cuando hablamos de un Centro de Control de Tierra, nos referimos al conjunto de hardware y software que unidos permiten la monitorización y/o control de vehículos autónomos. Debe incluir al menos una Interfaz Gráfica de Usuario (GUI) que permita monitorizar la información de telemetría (posición del vehículo en un mapa, ángulos de cabeceo, alabeo y guiñada, batería, velocidad, etc.), un equipo de comunicaciones para conectarse con los vehículos de forma remota y los elementos necesarios para enviar consignas de control. Además, dependiendo de la complejidad de la misión para la que haya sido diseñado y de los vehículos a monitorizar, puede incluir más o menos elementos adicionales que permitan visualizar la información de los sensores y actuadores que incorporen.

\subsection{ESTRUCTURA DEL CCT}

La estructura del centro de control de tierra que se describe en este artículo se basa en una arquitectura adaptativa dirigida por eventos (Adaptive Event-Driven Architecture) que ha sido adaptada para implementarlo, con el fin de superar los problemas derivados de la realización de misiones con equipos de vehículos autónomos heterogéneos reales y/o simulados que pueden cambiar según las necesidades de la misión. Para resolver los problemas de heterogeneidad en el equipo de dispositivos conectados, nuestro diseño arquitectónico combina ideas de las arquitecturas dirigidas a eventos (Event-Driven Architectures - EDAs) y del software auto-adaptativo, con módulos desacoplados que reaccionan ante los mensajes (eventos) recibi-

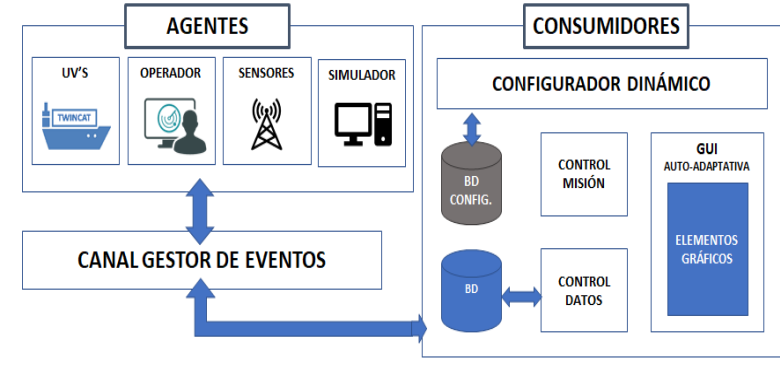

Figura 1: Esquema arquitectónico del CCT

dos, con el patron Observador (Observer pattern) que permite la implementación sencilla y robusta de un sistema de comunicaciones asíncrono. Una descripción detallada de esta arquitectura puede verse en [3].

Este entorno puede se usado para diseñar infraestructuras distribuidas complejas y adaptativas para aquellos ámbitos en los que se necesite integrar dispositivos heterogéneos (vehículos autónomos, sensores, agentes inteligentes autónomos, etc.), monitorizarlos y controlarlos.

Tal y como se muestra en la Figura 1, la arquitectura dirigida a eventos ayuda a desacoplar los agentes generadores de eventos (en este caso, sensores, vehículos, operadores, etc.) de los consumidores de eventos (en nuestro caso, la GUI del CCT) y permitirá recibir mensajes asíncronos en tiempo real de distintas procedencias de forma robusta y segura. Esto supone una gran ventaja a la hora de monitorizar/controlar un equipo de dispositivos heterogéneos que pueden enviar sus datos en cualquier instante de la misión. El desacoplamiento es también una característica muy importante para lograr la Adaptabilidad, ya que permite a cualquier infraestructura diseñada siguiendo el esquema modular añadir, quitar o cambiar en tiempo real cualquiera de los Consumidores o de los Agentes (sin importar su hardware, software o protocolo de comunicaciones).

Todo esto hace nuestro software tolerante a cambios en el entorno, los objetivos de la misión o la tarea actual, ya que permite introducir otros dispositivos, vehículos o sensores cuando la misión así lo requiera. Un ejemplo de la reconfiguración y control distribuido de varios vehículos ASV utilizando este CCT puede verse en [2], donde se muestra cómo se puede incorporar un nuevo equipo para controlar un ASV durante una misión. Además, el desacoplamiento potencia la simulación distribuida en tiempo real, permitiendo a una computadora remota generar datos, que modelan el comportamiento de un vehículo autónomo, y unirse al sistema en tiempo de ejecución. Esto nos permitirá verificar la eficacia de las ayudas introducidas 


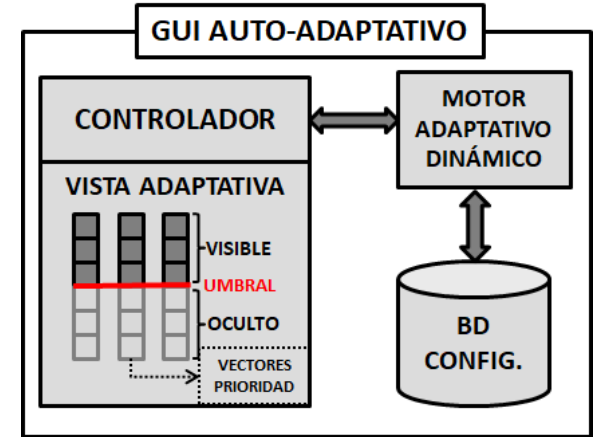

Figura 2: Esquema de la GUI Auto-adaptativa

sobre un conjunto de operadores, mediante el uso de un simulador de eventos que se conecta al mismo CCT que se utiliza con los vehículos reales.

Por último, los consumidores (representados en el lado derecho de la Figura 1) recogen la información de los agentes y la muestran al usuario, por lo que se pueden disponer de diferentes GUI según la finalidad del CCT. En nuestro caso, se ha diseñado una GUI auto-adaptativa que permite acondicionar la información que se muestra al usuario para reducir la carga de trabajo del operador y aumentar su rendimiento.

\subsection{GUI AUTO-ADAPTATIVA}

La interfaz gráfica de usuario (GUI) implementado es un software modular auto-adaptativo que reconfigura la vista del CCT en tiempo de ejecución con el objetivo de reducir la carga mental del operador. Una descripción más detallada del mismo puede encontrarse en [4].

Como se muestra en la Figura 2, consta de varios elementos. Por una parte, el motor adaptativo dinámico se encarga de monitorizar de forma contínua la conducta del operador durante la misión, estimar su carga de trabajo y calcular el umbral de transparencia. Por otra, el controlador es el ejecutor de los cambios dinámicos, es decir, aplica los cambios en los elementos gráficos solicitados por las políticas de transparencia y adaptabilidad marcadas por el motor adaptativo teniendo en cuenta también las directivas del usuario y de la misión. Además, la vista adaptativa es el módulo software que se ocupa de mostrar la información al operador (los elementos gráficos, el mapa, el comandador, etc.) y le permite interactuar con el resto del sistema. Finalmente, la base de datos incluye información de configuración para los otros módulos y almacena, por ejemplo, parámetros globales de la misión o las preferencias de cada operador para configurar la GUI y sus elementos gráficos (alarmas, datos de telemetría, nivel de batería, ... ).
El valor del umbral de transparencia es el que determina qué elementos de cada vehículo son mostrados en cada momento. Puede calcularse globalmente o particularizarse para cada vehículo, y depende del estado del operador y de la fase de la misión. En nuestro caso utilizaremos un valor igual para todos los vehículos independientemente de la fase de la misión. Su valor se puede estimar de múltiples formas: ad-hoc para una misión concreta, mediante algoritmos de inteligencia artificial o técnicas de aprendizaje máquina. Puesto que se ha diseñado un CCT con una arquitectura modular y desacoplada, este módulo puede cambiarse fácilmente por otros que implementen otros algoritmos para poder evaluar diferentes estrategias. En nuestro caso, con el fin de evaluar la viabilidad del CCT y su capacidad de disminuir la carga de trabajo del operador, utilizamos un sencillo árbol de decisión que viene representado por la Ecuación 1, donde $M_{u}$ indica si se debe modificar el valor actual del Umbral: si $M_{U}<0$ se incrementa en $\Delta U$, si $M_{U}>0$ se decrementa y si $M_{U}=0$ no se varía, donde $F_{s}(\cdot)$ representa a la función signo, $F_{f}(\cdot)$ a la función floor y $F_{s f}(\cdot)=F_{s}\left(F_{f}(\cdot)\right)$. Esta ecuación calcula el tipo de cambio con el que se debe modificar el umbral atendiendo a tres niveles. En el primer nivel se verifica si la carga de trabajo subjetiva $(C d T)$ sube o baja significativamente: si $C d T<33 \%$ se incrementa el Umbral y si $C d T>66 \%$ se baja. El segundo nivel actúa si CdT se encuentra en el intervalo intermedio (no varia significativamente) y tiene en cuenta el tiempo de reacción medido en los puntos de control $\left(T_{R}\right)$. Si el $T_{R}$ actual ha variado respecto al anterior más de un $45 \%$ (i.e. si $T_{R}<D T_{R}$, donde $\left.D T_{R}=0,45 \cdot T_{R a n t}\right)$ se sube el umbral y si está por encima del $45 \%$ se baja. Por último, en caso de que la variación del $T_{R}$ no sea significativa, la modificción del Umbral se llevará a cabo atendiendo a la variación del Tiempo Empleado $\left(T_{E}\right)$ de igual forma que con el $T_{R}$ (siendo $D T_{E}=0,45 \cdot T_{\text {Eant }}$ ). El valor de $\Delta U=1$ y el rango de los intervalos de cada uno de los parámetros monitorizados se han estimado a partir de la ejecución de diferentes misiones sobre un grupo de individuos diferentes a los del estudio que se indica en las secciones 3 y 4 .

$$
\begin{aligned}
M_{u} & =4 \cdot F_{f}\left(\frac{C d T-33}{33}\right) \\
& +2 \cdot F_{s}\left(T_{R}-\left(T_{\text {Rant }}-D T_{R}\right)\right) \cdot F_{s f}\left(\frac{\left|T_{R}-T_{\text {Rant }}\right|}{D T_{R}}\right) \\
& +F_{s}\left(T_{E}-\left(T_{\text {Eant }}-D T_{E}\right)\right) \cdot F_{s f}\left(\frac{\left|T_{E}-T_{\text {Eant }}\right|}{D T_{E}}\right)
\end{aligned}
$$

En la Figura 3 se muestra un ejemplo de la GUI del CCT cuando se realiza una misión con tres vehículos: dos vehículos autónomos de superficie (USV) y un vehículo autónomo aéreo (USV) de 


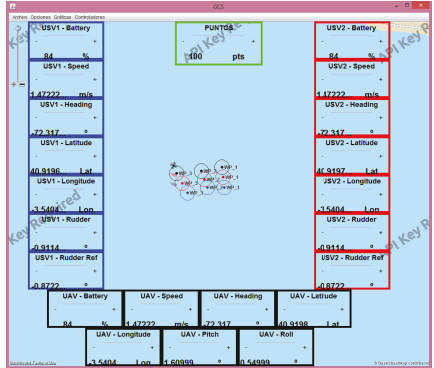

(a) GUI sin ayuda $(\mathrm{t}=100 \mathrm{~s})$

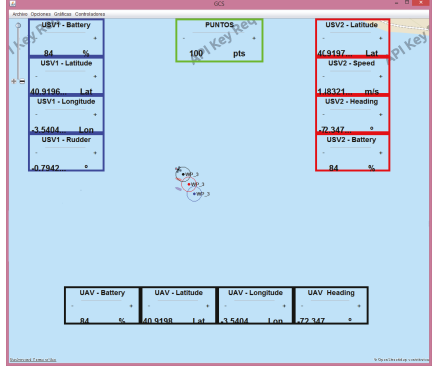

(b) GUI con ayuda $(\mathrm{t}=100 \mathrm{~s})$

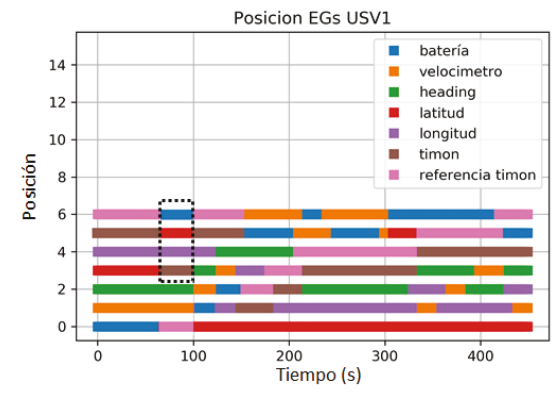

(c) Movimiento de los elementos del USV1

Figura 3: Interfaz de usuario (GUI)

apoyo. En la Figura 3a se muestra la GUI en un instante de la misión sin haber activado el motor adaptativo. En esta interfaz se muestran siempre los mismos elementos de los vehículos en la misma posición (dada por la configuración de la base de datos) a no ser que la modifique directamente el operador. Sin embargo, y tal y como se muestra la Figura 3b para el mismo instante de la misión que el de la Figura 3a, cuando actúa el motor adaptativo se muestran menos elementos al operador para ayudarle a localizar fácilmente la información de interés. Además, la adaptabilidad se ocupa de indicar qué elementos deberían aparecer cuando ocurre un determinado evento, por lo que cambian de posición o, aparecen y desaparecen. En la Figura 3c se muestra un ejemplo de la posición de los elementos gráficos del USV1 durante el desarrollo de la misión principal (que será explicada posteriormente) con ayudas al operador. También se encuadra la disposición de los elementos de USV1 en el instante mostrado en la Figura 3a. Considerando que los elementos con valor umbral inferior a 3 no son mostrados en la GUI, se puede comprobar que el nivel de la batería no es mostrado inicialmente al operador, aparece en la parte superior de la GUI en el instante analizado, posteriormente desaparece, y durante la mitad final de la misión aparece en la primera o segunda posición del GUI.

\section{EVALUACIÓN DE LA GUI AUTO-ADAPTATIVA}

Para evaluar la eficacia de la GUI auto-adaptativa se ha construido un simulador que emula las señales y sensores de los vehículos, y que es tratado como un agente más del CCT diseñado (constituyendo el agente SIMULADOR de la Figura 1).

Respecto a los criterios de evaluación, en [6] se recomienda, para una medición correcta de la carga mental de trabajo, una medida subjetiva a posteriori, otra de carga mental de trabajo de tareas primarias y una tercera de carga de trabajo de tareas secundarias, estas dos últimas realizadas durante la ejecución de la misión. Respecto al cuantificador subjetivo a posteriori, indicar que en [15] se comparan tres de las técnicas mas conocidas: el NASA-TLX, la Técnica de asesoramiento de carga de trabajo subjetiva (Subjective Workload Assesment Technique, SWAT) y el Perfil de carga de trabajo (Workload Profile, WP), y se elige el primero como mejor cuantificador para determinar el rendimiento de un individuo en una tarea particular. Respecto a los cuantificadores medidos durante la ejecución de la misión, en [16] se mencionan algunos directos (como consultar al operador su carga mental de trabajo subjetiva en un instante determinado de la misión) y otros indirectos (como la frecuencia de respuesta correcta, la tasa de error, o el tiempo de reacción en una tarea determinada).

En las secciones siguientes veremos cómo se han diseñado los experimentos y los elementos incluidos en el simulador para medir estos cuantificadores y, por tanto, evaluar la carga mental del operador durante la misión.

\subsection{ELEMENTOS DEL SIMULADOR}

Para la evaluación del efecto en el operador de la transparencia y la adaptabilidad se ha llevado a cabo una misión de monitorización en la que los operadores deben vigilar la correcta ejecución de la misión por parte de los vehículos autónomos y realizar ciertas actuaciones en caso de que se desvíen excesivamente de los puntos de paso (waypoints, WP) o estén en riesgo de colisión. Para que la misión sea la misma en todos los casos, y así se pueda comparar el resultado de los diferenres operadores, los vehículos pasan por los mismos waypoints (salvo un cierto ruido que puede desviarlos) y los eventos que ocurren son determinista (apareciendo en los mismos instantes de tiempo). Los eventos contemplados son ventanas emergentes que solicitan al operador una actuación o in- 
formación concreta. En particular, se consideran los siguientes:

- Eventos de verificación de misión correcta $(\mathrm{CK})$ : son comprobaciones de que el operador ha llevado a cabo ciertas acciones para garantizar que los vehículos funcionan adecuadamente. En concreto, son eventos de verificación de paso por el waypoint de una ruta (CK_Ruta) y de verificación del estado del vehículo (CK_Tim para el timón, CK_Vel para la velocidad y CK_Act para la actitud). Cuando ocurre el evento CK_Ruta el operador debe anotar la posición y velocidad del vehículo, y verificar si éste está suficientemente cerca del waypoint correspondiente, actuando para corregir su trayectoria en caso negativo; mientras que cuando ocurre uno de los eventos restantes el operador debe comparar y anotar el valor de referencia comandado frente al valor real.

- Alarmas (AL): en momentos puntuales pueden saltar alarmas sobre el nivel de batería, (AL_Bat) o la velocidad del vehículo (AL_Vel). Estos avisos deben ser atendidos cuanto antes, verificados y desactivados.

- Alerta de colisión (AL_COL): es el evento más urgente y debe ser atendido lo antes posible. Exige que el operador verifique ciertos valores (de velocidad y rumbo) del vehículo y tras una serie de cálculos, según el valor obtenido, que aplique una acción, que si es correcta evitará la colisión del vehículo y si no hará que el operador deba repetir el proceso.

Además de estos eventos de misión, se tomarán diferentes medidas de control, siguiendo la recomendación de [16], para seguir la evolución del operador durante la misión:

- Tiempo empleado en una tarea $\left(T_{E}\right)$, que es el tiempo que tarda el operador en realizar cada una de las acciones anteriores y que es calculado internamente por el simulador.

- Tiempo de reacción $\left(T_{R}\right)$, que es una medida de la capacidad del operador para realizar una tarea sencilla. Para medirlo, se le pide al operador que realice un cálculo matemático simple y que proporcione el valor del resultado. El tiempo que tarda en responder es un indicador de si está muy ocupado y mentalmente sobrecargado.

- Carga de trabajo subjetivo (CdT), que es una medida subjetiva, proporcionada por el operador, de su carga de trabajo. Para medirlo, se le preguntará cual es su carga de trabajo (valorada entre 1 y 100) que considera que se le exige en ese momento en la misión.

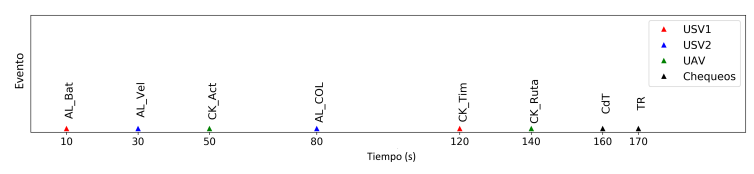

(a) Misión básica

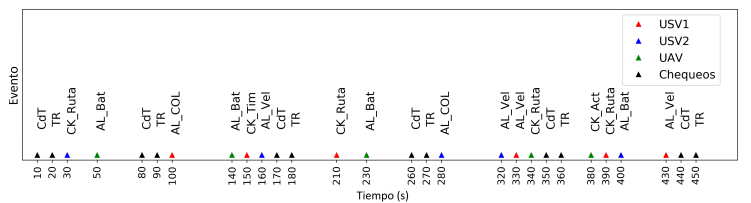

(b) Misión principal

Figura 4: Cronogramas de las misiones de test

Los dos últimos valores requieren que se introduzcan elementos (operaciones matemáticas y consultas de la carga de trabajo subjetiva) adicionales a la misión. Para asegurar que interfieren lo menos posible, sólo se incluyen en momentos puntuales para que puedan servir de referencia a la herramienta que evalúa la carga mental de trabajo.

\subsection{DISEÑO DEL EXPERIMENTO}

Los experimentos son individuales y sin comunicación entre operadores. Se realizan en dos fases.

En la primera fase se presenta la herramienta al operador, explicándole la finalidad del experimento, los elementos que aparecerán y cómo actuar con cada uno de ellos. A continuación, se realiza una misión corta y sencilla, donde aparecen una muestra de los elementos existentes (espaciados suficientemente) para que se familiaricen con la herramienta. En la Figura 4a se muestra un cronograma de la misión básica de entrenamiento donde aparece un único evento de cada tipo.

En la segunda fase, cuando los operadores ya están preparados, realizan la misión principal dos veces, una sin ningún tipo de ayuda y otra con ayudas de adaptabilidad y transparencia. Para poder comparar los resultados, los eventos, que se muestran en el cronograma de la Figura 4b, aparecen siempre en el mismo instante de tiempo. Además, al predefinir cuando ocurre cada evento se pueden simular distintas situaciones. De esta forma, se establecen tres etapas en la misión. La fase inicial (hasta los 50 segundos) sirve para establecer la base de tiempos y carga de trabajo de cada operador (está fresco y los eventos aparecen suficientemente espaciados para poder atenderlos adecuadamente). Es necesaria ya que cada operador es diferente respecto a su percepción de la carga de trabajo y a su tiempo de respuesta. En la etapa central (hasta los 400 s) los eventos se sitúan de modo que el operador tenga una carga de trabajo suficientemente alta para poder observar si las ayudas actúan ade- 


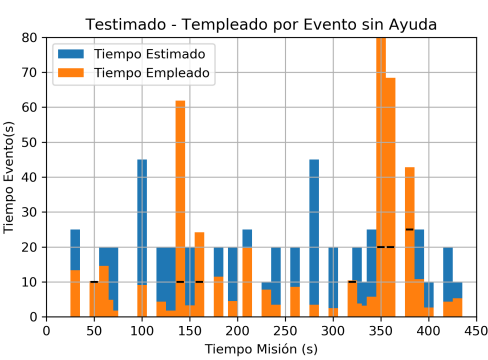

(a) Sin Ayuda

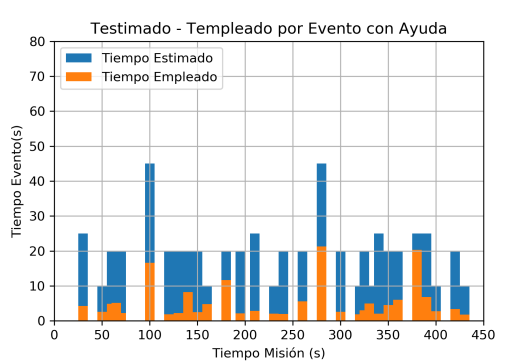

(b) Con Ayuda

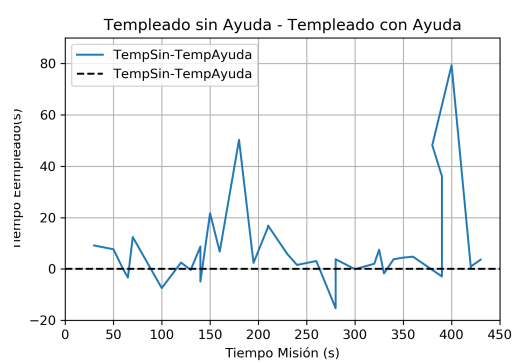

(c) Diferencia de tiempos empleados

Figura 5: Tiempo estimado y empleado por el usuario \#2 en realizar las tareas de la misión principal

cuadamente. Y en la etapa final (de $400 \mathrm{~s}$ hasta el final) los eventos vuelven a estar separados como al comienzo, con el objetivo de observar el estado mental y fatiga del operador al final de la misión.

Por último, los operadores realizan el test NASATXL para disponer de una medida a posteriori con la que contrastar/confirmar los resultados que se obtienen con la medidas en tiempo real.

Finalmente, indicar que todos estos datos son recogidos y procesados a posteriori, para poder realizar un análisis pormenorizado del comportamiento de cada operador.

\section{RESULTADOS}

Los experimentos y misiones indicados en la sección 3.2 se han llevado a cabo sobre un conjunto de 24 individuos (operadores). Para cada operador se recogen los cuantificadores presentado en la sección $3.1\left(\mathrm{CdT}, T_{R}\right.$ y $\left.T_{E}\right)$ y se analiza su evolución de forma individual.

A modo de ejemplo a continuación se presentan los resultados obtenidos por el operador \#2. En la figura 5 se muestra el tiempo empleado $\left(T_{E}\right)$ en realizar las tareas de los eventos de la misión principal (Figura 4b) frente al tiempo estimado que debería tardar. En la Figura 5a se muestra el tiempo empleado (comparado con el tiempo estimado en realizar la tarea) cuando ejecuta la misión sin ayuda y en la Figura 5b con ayuda. Como se puede ver, el tiempo en ejecutar las tareas correspondientes a los eventos señalados en el cronograma de la Figura 4b es menor en casi todos los casos cuando se proporciona ayuda al operador. En la Figura 5c se muestra la diferencia entre el tiempo empleado sin y con ayuda, por lo que valores positivos indican que se ha reducido el tiempo empleado cuando la ayuda está activa y valores elevados que la reducción es significativa.

Para el mismo operador, la Figura 6 muestra los eventos de control durante la misión. Señalar que la estima subjetiva de la carga de trabajo (CdT),

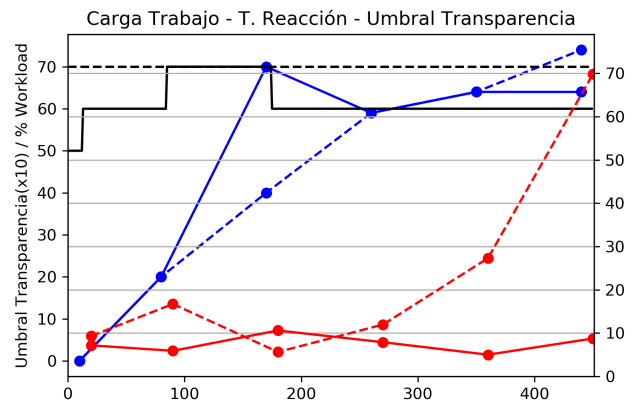

Figura 6: CdT, $T_{R}$ y Nivel de Transparencia con (líneas continuas) y sin (líneas discontinuas) ayuda

en azul, y tiempo de reacción $\left(T_{R}\right)$, en rojo, son habitualmente menores cuando hay ayuda al operador (líneas continuas) que cuando no las hay (líneas discontinuas). Se puede observar también que al bajar el nivel de transparencia (linea negra continua) se consigue que la carga de trabajo y el tiempo de respuesta dejen de subir, al contrario de lo que ocurre cuando no hay ayuda y se mantiene el umbral de transparencia fijo (línea negra discontinua). Esto es otro indicador de que las ayudas introducidas han logrado su objetivo.

Durante los experimentos se ha observado que tanto el tiempo de respuesta como la percepción que cada individuo tiene sobre su carga de trabajo puede variar mucho respecto a la de otros individuos. Por lo tanto, para mostrar los resultados globales de los individuos no se realizará la media de los valores individuales, sino de la respuesta relativa de cada individuo. Para ello se calcula la diferencia entre el Tiempo empleado sin ayuda y con ayuda (como se ha mostrado en la Figura 5c para el operador \#2), y se calcula la media, respecto a los diferentes operadores, de todos estos valores. Este valor medio se representa en la Figura 7 , donde se puede observar de nuevo que la mayoría de los valores son positivos, por lo que en general, las ayudas proporcionadas al operador permiten disminuir el tiempo que este necesita para atender los distintos eventos de la misión. 


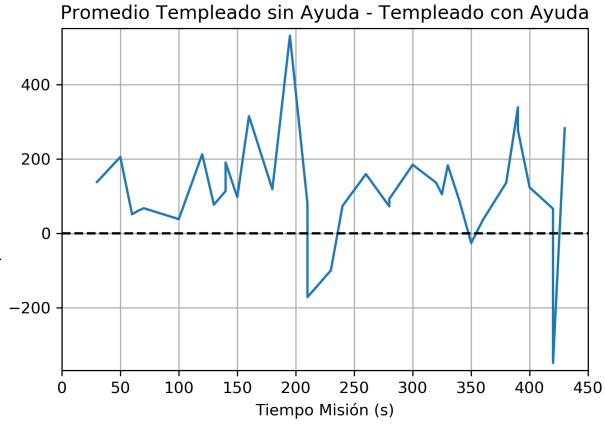

Figura 7: Promedio de la diferencia de tiempos empleados de todos los operadores

Por último, después de llevar a cabo ambas misiones, se ha realizado el test NASA-TLX como medida subjetiva a posteriori de la carga de trabajo de los operadores. Los resultados obtenidos se recogen en la Tabla 1. Según se especifica en [8], valores entre 900 y 1000 representan valores altos de carga mental de trabajo. A pesar de ello, se observa un menor valor en el test realizado después de la misión con ayuda que sin ayuda en la mayoría de los operadores, lo que señala una menor carga de trabajo. En la Figura 8 se representa la diferencia entre los indice NASA-TLX con ayuda y sin ayuda de todos los operadores, pudiéndose observar que en casi todos los operadores (salvo cinco) esta diferencia es positiva y que el grado de mejora es bastante significativo en algunos casos.

\section{CONCLUSIONES}

El diseño modular, escalable y configurable del CCT desarrollado facilita la inclusión de diversos módulos para personalizar y adaptar su funcionamiento fácilmente a distintos vehículos y requisitos. Esta ventaja ha sido aprovechada para construir una GUI adaptativa que, aplicando los conceptos de adaptabilidad y transparencia, modifica la información mostrada de cada vehículo, de modo que se reduzca y mejore el rendimiento del operador. El CCT se ha probado con éxito sobre un conjunto de operadores mostrando que la propuesta realizada es capaz de reducir la carga de trabajo del operador y su tiempo de respuesta.

El motor adaptativo actual, que se ocupa de ajustar el umbral de transparencia, utiliza un árbol de decisión. Aunque esto nos ha permitido probar que la propuesta permite reducir la carga mental de trabajo del operado, su comportamiento puede ser mejorado/ampliado para aumentar la eficacia de las ayudas al operador. Por ejemplo, el uso de técnicas de inteligencia artificial o de aprendizaje máquina en dicho motor permitirían identificar y adaptar la ayuda al tipo de misión y de opera-

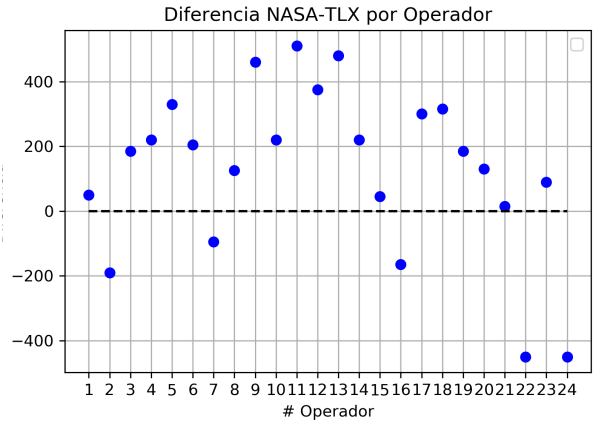

Figura 8: Mejora de carga de trabajo del operador (NASA-TLX)

dor, o prever un aumento de la carga mental del operador para poder actuar antes de que ésta se produzca.

\section{Agradecimientos}

Este trabajo ha sido financiado por el Ministerio de Educación, Cultura y Deporte bajo los proyectos DPI2013-46665-C2 y RTI2018-098962-B-C21.

English summary

\section{REDUCING OPERATORS MEN- TAL WORK LOAD IN AN ADAP- TIVE MULTI-VEHICLE CON- TROL STATION}

\section{Abstract}

The increasing number of autonomous vehicles, the growing complexity of the tasks involving them, and the amount of data to be considered makes it difficult for a single operator to monitor and control all the vehicles in the mission. Taking advantage of the modular, versatile, reconfigurable and multi-vehicle architectural design of the developed Ground Control Station $(G C T)$, we have incorporate in it aid mechanisms (adaptability and transparency) that lighten the displayed information and focus on the most relevant one for each situation within a mission, in order to reduce the operator mental workload. This article shows how these aids have been implemented and the experiments carried out on a set of operators to verify the GCT effectiveness in reducing the operators mental work load. 
Tabla 1: Resultados del NASA-TLX

\begin{tabular}{|c|l|l|l|l|l|l|l|l|l|l|l|l|}
\hline Misión & $\mathbf{\# 1}$ & $\mathbf{\# 2}$ & $\mathbf{\# 3}$ & $\mathbf{\# 4}$ & $\mathbf{\# 5}$ & $\mathbf{\# 6}$ & $\mathbf{\# 7}$ & $\mathbf{\# 8}$ & $\mathbf{\# 9}$ & $\mathbf{\# 1 0}$ & $\mathbf{\# 1 1}$ & $\mathbf{\# 1 2}$ \\
\hline Sin Ayuda & 850 & 710 & 1145 & 1260 & 1265 & 875 & 685 & 1175 & 910 & 730 & 1310 & 820 \\
\hline Con Ayuda & 800 & 900 & 960 & 1040 & 935 & 670 & 780 & 1050 & 450 & 510 & 800 & 445 \\
\hline \hline Misión & $\mathbf{\# 1 3}$ & $\mathbf{\# 1 4}$ & $\mathbf{\# 1 5}$ & $\mathbf{\# 1 6}$ & $\mathbf{\# 1 7}$ & $\mathbf{\# 1 8}$ & $\mathbf{\# 1 9}$ & $\mathbf{\# 2 0}$ & $\mathbf{\# 2 1}$ & $\mathbf{\# 2 2}$ & $\mathbf{\# 2 3}$ & $\mathbf{\# 2 4}$ \\
\hline Sin Ayuda & 910 & 995 & 820 & 850 & 935 & 885 & 1415 & 1205 & 990 & 425 & 1130 & 475 \\
\hline Con Ayuda & 430 & 775 & 775 & 1015 & 635 & 570 & 1230 & 1075 & 975 & 875 & 1040 & 925 \\
\hline
\end{tabular}

Keywords: Mental work load, Control stations, Adaptive software interfaces.

\section{Referencias}

[1] M. Amoui, M. Derakhshanmanesh, J. Ebert, and L. Tahvildaria. Achieving dynamic adaptation via management and interpretation of runtime models. Journal of Systems and Software, 2012.

[2] J.A. Bonache-Seco, J. Dormido-Canto, M. Montalvo, J.A. López-Orozco, E. BesadaPortas, and J.M. de la Cruz. Centro de control de tierra para colaboración de vehículos autónomos marinos. Revista Iberoamericana de Automática e Informática Industrial, 2017.

[3] J.A. Bonache-Seco, J.A. Lopez-Orozco, E. Besada-Portas, and J.L. Risco-Martín. Adaptive event driven framework for real time multi-agent missions. International Symposium on Distributed Simulation and Real-Time Applications, 2018.

[4] J.A. Bonache-Seco, J.A. López-Orozco, E. Besada-Portas, and J.L. Risco-Martín. ART-GCS: An adaptive real-time multiagent ground control station. In Spring Simulation Conference, May 2019.

[5] J. Chen, K. Procci, M. Boyce, J. Wright, A. Garcia, and M. Barnes. Situation awareness-based agent transparency, 2014.

[6] D. de Waard. The Measurement of Drivers Mental Workload, volume 1. Groningen University, Traffic Research Centre, 1996.

[7] L. Filipponi, A. Vitaletti, G. Landi, V. Memeo, G. Laura, and P. Pucci. Smart city: An event driven architecture for monitoring public spaces with heterogeneous sensors. In International Conference on Sensor Technologies and Applications, 2010.

[8] S. Hart. Nasa-task load index (NASA-TLX); 20 years later. Human Factors and Ergonomics Society Annual Meeting, 50, 2006.
[9] T. Helldin. Transparency for future semiautomated systems. PhD thesis, Orebro University, 2014.

[10] J. Heo, S. Kim, and Y. James Kwon. Design of ground control station for operation of multiple combat entities. Journal of Computer and Communications, 04, 012016.

[11] M. Jovanovic and D. Starcevic. Software architecture for ground control station for unmanned aerial vehicle. EUROMICRO Conference on Software Engineering and Advanced Applications, 2010.

[12] J.E. Mercado, M.A. Rupp, J.Y.C. Chen, M.J. Barnes, Daniel Barber, and Katelyn Procci. Intelligent agent transparency in humanagent teaming for multi- $\mathrm{UxV}$ management. Human Factors, 58(3), 2016.

[13] D. Perez, I. Maza, F. Caballero, D. Scarlatti, E. Casado, and A. Ollero. A ground control station for a multi-uav surveillance system. Journal of Intelligent \& Robotic Systems, 69(1), 2013.

[14] QGroundControl. A UAV Control Station. [Online] http://qgroundcontrol.com/, 2017. Accedido en Marzo de 2017.

[15] S. Rubio, E. Díaz, J. Martín, and J. Puente. Evaluation of subjective mental workload: A comparison of SWAT, NASA-TLX, and Workload Profile Methods. Applied Psychology, $53,2004$.

[16] O. Sebastián and M. delHoyo. La carga mental de trabajo, pages 1-51. Instituto Nacional de Seguridad e Higiene en el Trabajo, 2004.

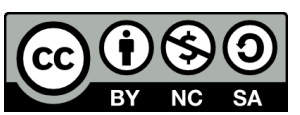
(C) 2019 by the authors. Submitted for possible open access publication under the terms and conditions of the Creative Commons Attribution CC BY-NC-SA 4.0 license (https://creativecommons.org/licenses/by-ncsa/4.0/deed.es). 